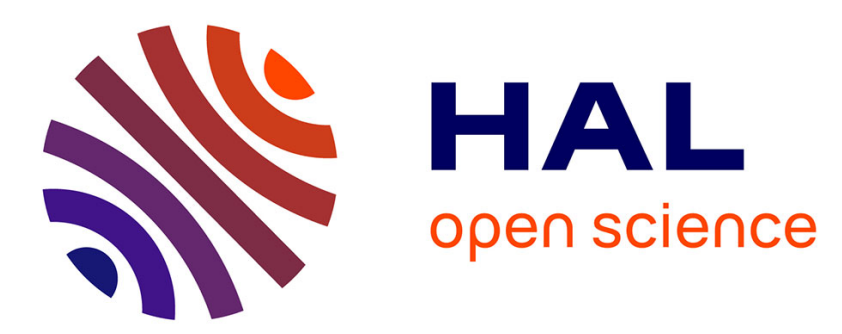

\title{
Ultrasonic self-focusing and self-transparency in the liquid
}

\author{
G. Lyakhov, K. Shipilov, O. Umnova
}

\section{To cite this version:}

G. Lyakhov, K. Shipilov, O. Umnova. Ultrasonic self-focusing and self-transparency in the liquid. Journal de Physique IV Proceedings, 1994, 04 (C5), pp.C5-1201-C5-1204. 10.1051/jp4:19945265 . jpa-00252955

\section{HAL Id: jpa-00252955 https://hal.science/jpa-00252955}

Submitted on 1 Jan 1994

HAL is a multi-disciplinary open access archive for the deposit and dissemination of scientific research documents, whether they are published or not. The documents may come from teaching and research institutions in France or abroad, or from public or private research centers.
L'archive ouverte pluridisciplinaire HAL, est destinée au dépôt et à la diffusion de documents scientifiques de niveau recherche, publiés ou non, émanant des établissements d'enseignement et de recherche français ou étrangers, des laboratoires publics ou privés. 


\title{
Ultrasonic self-focusing and self-transparency in the liquid
}

\author{
G.A. LYAKHOV, K.F. SHIPILOV and O.V. UMNOVA
}

General Physics Institute, Russian Academy of Sciences, Vavilov str. 38, 117924 Moscow, Russia

\begin{abstract}
Process of thermal self -action of powerful ultrasonic signals in viscous liquids are theoretically and experimentally investigated. Simultaneous realization of acoustic beam self-focusing and sound induced self--transparency of liquid gives a new nonlinear effect of high energy wave packet self-concentration. A pulse with duration $\Delta t \sim 10 \mathrm{~s}$ is formed inside the liquid, the intensity of ultrasound in its peak is several times greater than the input level. The parameters of this process are calculated, which are in a good agreement with experimental data.
\end{abstract}

\section{Introduction}

Estimates [1] have shown, that only thermal mechanism of actual liquid nonlinearity can distinguish in nonexotic liquids, ultrasonic beam self - focusing (SF) in the background of a shock wave formation in a strong field and of other competitive processes, such as sound induced longitudinal streams and convection, as well as diffusion heat transport. This estimates yielded the conditions for experimental realization of thermal SF of ultrasound. For the first time this phenomenon was observed in relatively flowable benzene [2] and then in more viscous glycerine [3]; the results of these experiments are in good agreement with theoretical estimates. In glycerine, together with SF, an effect of acoustic self - transparency (ST) was observed, i. e. an increase in depth of ultrasound penetration into medium with the growth of input acoustic energy. ST is stimulated by the temperature dependence of absorptance $\frac{\partial \delta}{\partial T}<0$ noticeable in glycerine even at room temperature. Upon transition into the relaxation range (i. e. $-70 \div-30^{\circ} \mathrm{C}$ ) temperature derivatives of both the absorptance and sound velocity $\mathrm{c}$ increase considerably (fig.1). That is why the combines influence of SF and ST must be taken into account in analysis of ultrasonic self - action in the relaxation region of temperatures.

\section{Calculation of parameters of wave packet self - con- centration in liquids}

Keeping in mind a general description of self-action including ST near the relaxation region, the explicit dependence of absorption coefficient $\delta$ on sound-induced change of temperature 
$T$ must be incorporated in the parabolic approximation equations: $\delta=\delta_{0}-|\partial \delta / \partial T| T$. The equation for $T$ and sound pressure $p$ are :

$$
\begin{gathered}
\partial_{t} T=\left[\delta_{0}-|\partial \delta / \partial T|\right]|p|^{2} /\left(\rho c_{0} c_{p}\right) \\
\left(\partial_{x}+c_{0}^{-1} \partial_{t}+i\left(c_{0} / 2 \omega\right) \nabla_{\perp}^{2}\right) p=-\left[\delta_{0}-(\partial \delta / \partial T) T\right] p+ \\
+\imath\left(\omega / c_{0}^{2}\right)|\partial c / \partial T| T p
\end{gathered}
$$

The thermal conductivity process neglected considering that the time of thermal smearing $\tau_{T}=a^{2} / \xi=10^{2} s(a=0,5 \mathrm{~cm}$ - radius of the beam) exceeds characteristic times of $\mathrm{SF}$ and ST. For a beam of finite width and the use of near - axis approximation the solutions of (1) are in the form (assume the gaussian profile at the input:

$$
\begin{gathered}
I=p^{2} / 2 \rho c_{0}=I_{0} J(t, x) \exp \left(-R(t, x) \tilde{r^{2}}\right) \\
-\left(c_{0} / \omega a^{2} \delta_{0}\right) \arg p=\Phi(t, x)+\Psi(t, x) \tilde{r^{2}} / 2 \\
1-\left|\frac{\partial \delta}{\partial T} \frac{T}{\delta_{0}}\right|=\Theta(t, x)+Y(t, x) \tilde{r^{2}} / 2 \\
\tilde{r}=r / a
\end{gathered}
$$

In coordinates $u=\delta_{0} x, v=\delta_{0}\left(c_{0} t-x\right)$ from (1) it follows that:

$$
\begin{gathered}
\partial_{u} J+2 \Psi J=-\Theta J, \partial_{u} \Psi+\Psi^{2}=-b_{1} J+b_{2} R^{2} \\
\partial_{u} R+2 \Psi R=Y / 2, \partial_{v} \Theta=b_{3} J \Theta \\
\partial_{v} Y=-b_{3} J Y+2 b_{3} J \Theta R, \partial_{u} \Phi=b_{1}(1-\Theta)-b_{2} R
\end{gathered}
$$

The efficiency of the process is defined by parameters $b_{1}-b_{3}$. We estimate their values, as well as all subsequent estimates are performed, for glycerine at $T_{0}=-50^{\circ} \mathrm{C}$ :

$$
\begin{gathered}
b_{1}=|\partial(\ln c)| / \partial T /\left[|\partial \delta / \partial T| \delta_{0} a^{2}\right] \approx 10^{-2} \\
b_{2}=\left(k^{2} a^{4} \delta_{0}^{2}\right)^{-3} \approx 3 \cdot 10^{-5} \\
b_{3}=|\partial \delta / \partial T| I_{0} /\left(\delta_{0} \rho c_{p} c_{0}\right) \approx 10^{-5} \div 10^{-7}
\end{gathered}
$$

Boundary conditions for (3) are: at $u=0: v=\delta_{0} c_{0} t: J=R=1: \Psi=\Pi=0$ at $v=0: \Theta=0 Y=1$.

The analytical solution of (3), derived by iterative process, gives:

$$
\begin{aligned}
& J \simeq 2 J_{0} /\left[\cos (2 Q)+\cosh \left(b_{2}^{1 / 2} Q / b_{1}\right)\right] \\
& R \simeq 2 R_{0} /\left[\cos (2 Q)+\cosh \left(b_{2}^{1 / 2} Q / b_{1}\right)\right]
\end{aligned}
$$

where $J_{0}=\exp (\xi) /\left[\exp (\xi)+\exp \left(\delta_{0} x\right)-1\right], R_{0}=1+\left\{\xi\left[\exp \left(\delta_{0} x\right)-1\right]\right\} /\left[\exp (\xi)+\exp \left(\delta_{0} x\right)-\right.$ 1], $\xi=I_{0}|\partial \delta / \partial T|\left(t-x / c_{0}\right) /\left(\rho c_{p}\right)$

If $\xi \gg 1$, then $Q\left(\xi, \delta_{0} x\right) \sim 2^{3 / 2}\left(b_{1} \xi\right)^{1 / 2} \arctan \left\{\exp \left[\left(\delta_{0} x-\xi\right) / 2\right]\right.$. 


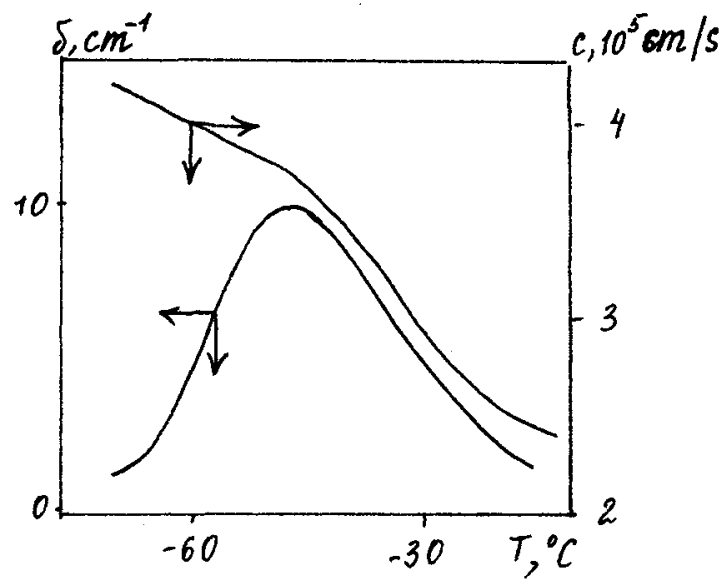

Figure 1: Temperature dependence of sound velocity $c$, and absorptance $\delta$ in glycerine, sound frequency $\omega / 2 \pi=2 \mathrm{MHz}$

In viscous liquid it is reasonable to consider $J$ only for $\xi \sim \delta_{0} x \gg 1$, while for smaller $\xi$ the signal is closed to zero. The intensity $J$ at the beam axis increases with time $\xi$ until the distance covered in liquid $x<x_{0} ; x_{0}$ is defined by:

$$
\left(2^{5} b_{1} \delta_{0} x_{0}\right)^{1 / 2} \arctan \left[\left(\delta_{0} x_{0}\right)^{1 / 2}\right]=\pi
$$

in glycerine $x_{0} \simeq 1.5 \mathrm{~cm}$. At larger distances $x>x_{0}$ the pulse structure is formed: peaks arise at a finite number of points, the coordinates $\xi$ of which are defined by;

$$
\left(2^{5} b_{1} \xi\right)^{1 / 2} \arctan \left[\exp \left(\delta_{0} x-\xi\right) / 2\right]=\pi(2 n-1)
$$

The peak values of $J$ and $R$ are:

$$
\begin{aligned}
& J_{p} \simeq 16 b_{1}^{2} J_{0 p} /\left(\pi^{2}(2 n-1)^{2} b_{2}\right) \\
& R_{p} \simeq 16 b_{1}^{2} R_{0 p} /\left(\pi^{2}(2 n-1)^{2} b_{1}\right)
\end{aligned}
$$

If the distance from the transducers is $x=7.5 \mathrm{~cm}$ the intensity of the first peak is $J(\xi=15) \ll$ 1 that at the second peak is $J(\xi \simeq 62)=3$. The relative radius of the beam $r \simeq R^{-1 / 2} \simeq 0.2$. The duration of the pulse formed is: $\Delta \xi \simeq 2 \pi /\left(2 b_{1} \delta_{0} x\right)^{1 / 2} \simeq 6$. Turning to real time $t$, we find, that for input intensity $J_{0}=8 \mathrm{~W} / \mathrm{cm}^{2}, 62 \mathrm{~s}$ after switching on the source, a pulse of duration $\Delta t \simeq 6 s$ is formed. Its intensity is 3 times higher, than the input evel. The distribution of intensity $J$ along the axis $x$ foci at $x_{n}=\xi / \delta_{0}+2 \ln [G(\xi, n)]$, where $G(\xi, n)=$ $\tan \left[\pi(2 n-1) 2^{-5 / 2}\left(b_{1} \xi\right)^{-1 / 2}\right]$. The dependence of SF length $L-f$ on energy $W=\pi a^{2} I_{0} t$ has a minimum $L_{f}=x_{0}$ when $W=W^{0}=\pi a^{2} \rho_{0} c_{p}\left[\delta_{0} x_{0}-\ln \left(\delta_{0} x_{0}\right)\right] /|\partial \delta / \partial T| \simeq 200 J$. The dependence $J(t, x)$ is presented in fig. 2 .

Experimental realizations of self - concentration process demonstrates a good agreement with theoretical results if the input intensity of ultrasound is in the range $5<I<10 \mathrm{~W} / \mathrm{cm}^{2}$. The maximum level of intensity $10^{2} \mathrm{~W} / \mathrm{cm}^{2}$ was reached experimentally at $T_{0}=-54^{0} \mathrm{C}, I_{0} \simeq$ $10 \mathrm{~W} / \mathrm{cm}^{2}$. 


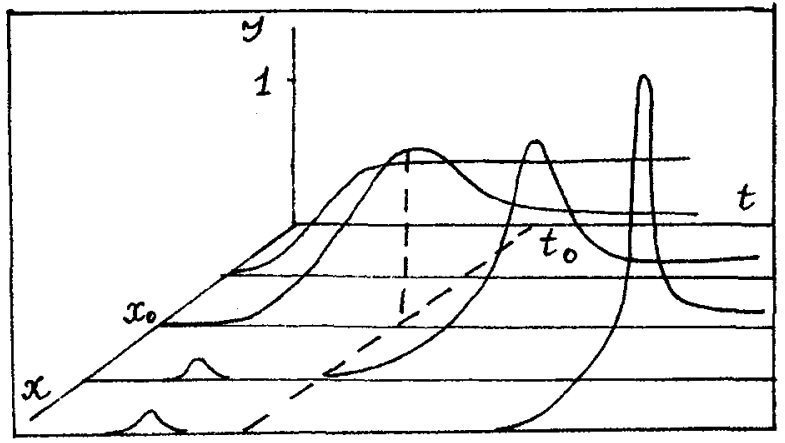

Figure 2: Acoustic beam self-focusing and self-transparency in liquid

\section{Conclusions.}

The phenomenon of acoustic energy self - concentration is perspective for applications. The large growth of intensity, in comparison with the input level, permits a strong and controlled ultrasound in deep layers of matter without noticeable influence on its surface. This feature can be employed in medicine and technology.

\section{References}

[1] F.V. Bunkin, K.J.Volyak, G.A.Lyakhov, Sov.Phys. JETP 2 (1982) p.316-321.

[2] V.A.Assman, F.V.Bunkin, G.A.Lyakhov, et al. Sov.Phys JETP lett. 41 (1985) p.102-104.

[3] V.A.Assman, F.V.Bunkin, G.A.Lyakhov, et al. Sov.Phys. Acoustics 31 (1985) p.182-184 